\title{
La Seguridad en la Producción de Alimentos en la Granja: Buenas Prácticas Agrícolas y Buenas Prácticas de Manejo - Sanidad en las Instalaciones de Empaque ${ }^{1}$
}

\author{
Federico G. Caro, Alexandra Chang, Renée Goodrich-Schneider, y Keith R. Schneider ${ }^{2}$
}

\section{Introducción}

Las Buenas Prácticas Agrícolas (BPA) y las Buenas Prácticas de Manejo (BPM) abarcan los procedimientos generales que los productores, empacadores y procesadores de frutas y verduras frescas deben seguir para garantizar la seguridad de sus productos. Las BPA son usadas antes de la cosecha (es decir, en el campo), mientras que las BPM se utilizan luego de la cosecha, incluyendo el empaque y envío. Esta hoja informativa aborda las BPA relacionadas al saneamiento de instalaciones de empaque. Hay otras siete hojas informativas de la Extensión Cooperativa de la Florida acerca de la "Inocuidad de los Alimentos en la Granja." Esta serie se centra en aspectos específicos del programa de BPA y cómo se relacionan con los cultivos y las prácticas de la Florida.

\section{Riesgos Microbianos}

La falta de higiene en las instalaciones de empaque aumenta en gran medida el riesgo de la contaminación de productos frescos. Todo lo que entra en contacto con los productos puede ser una fuente de patógenos, desde el equipo utilizado hasta el suministro de agua. Para reducir el potencial de contaminación, es importante adoptar un papel proactivo manteniendo estándares altos de saneamiento en el proceso de empaque.

\section{Reglas Generales}

El Estatuto 21 del Código de Regulaciones Federales, Parte 110, sección 20 describe las BPA para las instalaciones y los terrenos de las plantas productoras. Se incluyen los requisitos para el almacenamiento adecuado de los equipos, la sanidad de instalaciones y el control de plagas (1). Florida ha adoptado requisitos similares para las prácticas postcosecha en el capítulo 5 K-4 en el Código Administrativo de la Florida (2). Para los productores de tomate de la Florida, el Departamento de Agricultura y Servicios al Consumidor ha implementado las Buenas Prácticas Agrícolas del Tomate (T-BPA) y Buenas Prácticas de Manejo del Tomate (T-BPM) en Julio del 2008 con el propósito de mejorar la seguridad de los tomates en todas las etapas de producción (3). Encontrará mas información acerca del saneamiento en plantas de empaque en el Manual de Buenas Prácticas del Tomate (4).

En respuesta y reconocimiento de los problemas crecientes de seguridad alimentaria, la Ley de Modernización Para la Inocuidad de los Alimentos fue aprobada por el Congreso

1. The English version of this document is FSHN12-05/FS189 Food Safety on the Farm: Good Agricultural Practices and Good Handling Practices Packing Facility Sanitation. Este documento es FSHN12-05s, uno de una serie del Departamento de Ciencia de los Alimentos y Nutrición Humana, Servicio de Extensión Cooperativa, IFAS de la Universidad de Florida. Publicación: febrero de 2013. Ciencia de los Alimentos y Nutrición Humana, Servicio de Extensión Cooperativa, IFAS de la Universidad de Florida, Gainesville, FL 32611-0370. Por favor, visite nuestro sitio web EDIS en http://edis. ifas.ufl.edu/.

2. F. G. Caro, BS., técnico; A. Chang, estudiante de posgrado; R. M. Goodrich-Schneider, PhD, profesora asociada, y K. R. Schneider, PhD, profesor asociado; todos del Depto. de Ciencia de los Alimentos y Nutrición Humana, Servicio de Extensión Cooperativa, IFAS de la Universidad de Florida, Gainesville, FL 32611-0370. 
y firmada por el Presidente en Enero del 2011. La nueva ley exige a las empresas implementar un programa de seguridad alimentaria que minimice significativamente los riesgos asociados con enfermedades transmitidas por alimentos. Tomar medidas inmediatas para aplicar buenas prácticas agrícolas para mantener condiciones sanitarias adecuadas en las empacadoras beneficia a las empresas y mejora la seguridad de los productos en general.

\section{Como Controlar Riesgos Potenciales}

Las BPA son fundamentales para reducir los riesgos potenciales de contaminación y proteger a los consumidores contra las enfermedades transmitidas por los alimentos. De todas las BPA que pueden ser implementadas para minimizar la contaminación de los productos, las siguientes han sido recomendadas por la Administración de Alimentos y Fármacos (FDA) para el empaque de productos, mantenimiento de las instalaciones y control de plagas (5).

\section{Reglas Generales de Embalaje}

Como parte de las BPA generales de embalaje, los trabajadores involucrados en el manejo de productos frescos deben practicar buena higiene. Para obtener más información, vea "Seguridad de los Alimentos de la Granja: Buenas Prácticas Agrícolas y Buenas Prácticas de Manejo - Salud e higiene del trabajador," una ficha técnica de esta serie por Schneider y otros (6).

- Quite la mayor cantidad de tierra y barro como sea posible de los productos frescos antes de moverlos hacia las instalaciones de empaque - los productos pueden estar expuestos a estiércol y materia fecal en el suelo. La eliminación de la suciedad de la planta empacadora reduce al mínimo la acumulación de patógenos potenciales dentro de las instalaciones.

- Como parte del proceso de selección y clasificación, separe los productos dañados del resto de la cosecha para evitar la posible propagación de patógenos a los productos en buenas condiciones.

- Tome medidas para evitar que los contaminantes transportados por el aire entren a la empacadora - Es importante situar las instalaciones lejos del ganado, aves de corral o áreas de almacenamiento de estiércol, ya que pueden ser una fuente de contaminantes en el aire.
- Reparar o descartar envases deteriorados e inspeccionarlos con regularidad. Si están dañados y no pueden ser limpiados a fondo deben ser desechados.

- Limpie y desinfecte las paletas, contenedores y recipientes antes de usarlos para transportar frutas y verduras frescas - Tenga cuidado de no producir contaminación cruzada mediante la exposición de los contenedores a la tierra y el estiércol.

- Proteja los contenedores limpios o nuevos contra la contaminación durante el almacenamiento. Algunas fuentes de contaminantes que pueden afectar estos son las plagas, la suciedad y la condensación generada por equipos y estructuras que se encuentran por encima de los contenedores en el área de almacenamiento.

\section{Consideraciones generales para el Mantenimiento de las Instalaciones}

Las frutas y verduras frescas son muy susceptibles a la contaminación durante el manejo pos-cosecha debido al contacto frecuente con el agua de lavado y las superficies del equipo. El agua recirculada puede transferir agentes patógenos a los cultivos, y por lo tanto debe ser desinfectada de forma continua. La limpieza habitual de las plantas de embalaje y almacenamiento ayuda a prevenir la contaminación. El equipo utilizado en la selección, clasificación y envasado de los productos debe ser diseñado de modo que pueda ser limpiado adecuadamente. Las siguientes BPA deben ser observadas en el saneamiento de las instalaciones de embalaje.

- La calidad del agua utilizada debe ser monitoreada a través de análisis y estudios. Cambie el agua cuando sea necesario, y desinfecte las superficies que entren en contacto con el agua. Para más información, refiérase a "Inocuidad de los Alimentos en la Granja: Buenas Prácticas Agrícolas y Buenas Prácticas de Manejo - El Agua," una hoja informativa de esta serie por Schneider y otros (7).

- Se deben lavar todos los equipos y ropa de protección, tales como delantales y guantes, ya que pueden acumular polvo y patógenos a través del tiempo. Por lo tanto se deben limpiar regularmente, inspeccionar y detectar defectos, y reemplazarlos si están dañados.

- Limpie las áreas de empaque al fin de cada día; la eliminación de lodo y escombros es importante. Se deben desinfectar las áreas de lavado, clasificación, selección y 
empaque lo cual minimiza la posibilidad de supervivencia y crecimiento microbiano.

- Inspeccione el sistema de refrigeración diariamente para asegurar el funcionamiento adecuado del equipo y manténgalo limpio.

- Limpie las áreas de almacenamiento regularmente. La remoción de escombros, de otras cosas guardadas que no sean necesarias y la limpieza periódica de estas áreas pueden ayudar a prevenir la contaminación. Se deben tomar medidas para minimizar el polvo y otros contaminantes en el aire.

\section{Control de Plagas}

Los animales pueden ser vectores de enfermedades ya que transportan y transfieren microorganismos patógenos cuando entran en contacto con la cosecha. Excluya a las mascotas en toda la planta de empaque. Para reducir al mínimo la posibilidad de contaminación, se deben tomar precauciones contra una variedad de diferentes plagas, incluyendo mamíferos, aves, caracoles, reptiles e insectos. Considere las siguientes sugerencias.

- Establezca un sistema de control de plagas - Un programa independiente de control de plagas implementado por operadores con licencia, forma parte del sistema general de inocuidad de los alimentos. Puede ayudar a reducir el riesgo de contaminación al exigir un monitoreo frecuente y la limpieza de las zonas afectadas en todas las instalaciones. Haga un seguimiento con la persona a cargo del control de plagas para garantizar que las BPA se estén siguiendo y que el proceso esté funcionando.

- Conserve el lugar en buenas condiciones - Impida que las plagas habiten, se alimenten y reproduzcan alrededor de los campos de producción. Mantenga los terrenos libres de desechos, basura y otros elementos innecesarios, tales como equipos viejos y sin uso. Corte el pasto a menudo, y mantenga un desagüe eficiente para evitar el agua estancada y prevenir el estrés de las plantas.

- Monitorear y dar mantenimiento a las instalaciones a menudo - Como parte de un programa de control de plagas, revise regularmente todas las instalaciones buscando evidencia de plagas. Mantenga limpias las instalaciones mediante la eliminación inmediata de animales muertos, la limpieza de las superficies sucias, y tratando de reducir al mínimo los posibles agujeros o madrigueras donde las plagas pueden ocultarse o hacer nidos.
- Considere bloquear el acceso de plagas en instalaciones cerradas - Sellar los agujeros en las paredes, puertas, pisos, aberturas, etc. que las plagas pueden utilizar para entrar en la instalación. Utilice alambrados, cortinas, y hasta trampas cuando sea necesario.

- Utilice un registro de control de plagas - Mantenga un registro de las fechas de inspección y de lo que se ha hecho para solucionar los problemas con las plagas. Se deben monitorear las áreas afectadas y tratadas a menudo para determinar si otros métodos más eficaces deberían ser usados.

\section{Referencias}

1. Código de Regulaciones Federales. 2010a. Buenas Prácticas de Manufactura en la fabricación, el embalaje, o almacenamiento de alimentos: las plantas y campos. Título 21, Parte 110.20. Washington, DC: EE.UU. Administración de Alimentos y Fármacos (FDA), la Oficina del Registro Federal.

2. Código Administrativo de la Florida. 2004. Regla 5K-4.004. Requisitos generales para la fabricación, el procesamiento, el embalaje, almacenamiento y venta de alimentos. Disponible en: https://www.flrules.org/ gateway/chapterhome.asp? chapter=5k-4. Consultado el 10 de marzo del 2011.

3. Código Administrativo de la Florida. 2007. Regla 5 G-6. Inspección del Tomate. Disponible en: https://www. flrules.org/gateway/chapterhome. asp? chapter $=5 \mathrm{~g}-6$. Consultado el 10 de marzo del 2011.

4. Departamento de Agricultura y Servicios al Consumidor. 2007. Manual de Buenas Prácticas del Tomate. Tallahassee, FL: Departamento de Agricultura y Servicios al Consumidor de la División de Seguridad Alimentaria de la Florida. Disponible en: http://www.doacs.state.fl.us/fs/ TomatoBestPractices.pdf. Consultado el 10 de marzo del 2011.

5. Administración de Alimentos y Fármacos (FDA) .1998. Guía para reducir al mínimo el riesgo microbiano en frutas y verduras frescas. Washington, DC: EE.UU. El Departamento de Salud y Servicios Humanos de la FDA. Disponible en: http://www.fda.gov/downloads/Food/ GuidanceComplianceRegulatoryInformation/GuidanceDocuments/ProduceandPlanProducts/UCM169112. pdf Consultado el 10 de marzo del 2011. 
6. Schneider, KR., R. Goodrich Schneider, A. Chang. 2009. Inocuidad de los alimentos en la granja: las buenas prácticas agrícolas y buenas prácticas de manejo - la salud de los trabajadores y su higiene. Gainesville, FL: Departamento de Ciencia de los Alimentos y Nutrición Humana, Servicio de Extensión Cooperativa, IFAS de la Universidad de Florida. Disponible en: http://edis.ifas.ufl. edu.

7. Schneider, KR., R. Goodrich Schneider, DL. Archer. 2006. Inocuidad de los alimentos en la granja: las buenas prácticas agrícolas y buenas prácticas de manejo - el agua. Documento FSHN06-02. Gainesville, FL: Departamento de Ciencia de los Alimentos y Nutrición Humana, Servicio de Extensión Cooperativa, IFAS de la Universidad de Florida. Disponible en: http://edis.ifas.ufl.edu/FS136. Consultado el 10 de marzo del 2011. 